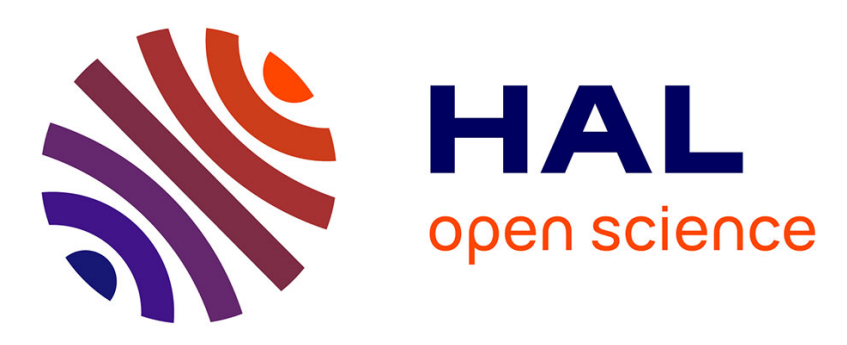

\title{
Determination of the melting curve of gold up to 110 GPa
}

Gunnar Weck, Vanina Recoules, Jean-Antoine Queyroux, Frédéric Datchi, Johann Bouchet, Sandra Ninet, Gaston Garbarino, Mohamed Mezouar, Paul Loubeyre

\section{To cite this version:}

Gunnar Weck, Vanina Recoules, Jean-Antoine Queyroux, Frédéric Datchi, Johann Bouchet, et al.. Determination of the melting curve of gold up to $110 \mathrm{GPa}$. Physical Review B: Condensed Matter and Materials Physics (1998-2015), 2020, 101, 10.1103/PhysRevB.101.014106 . hal-02468892

\section{HAL Id: hal-02468892 https://hal.science/hal-02468892}

Submitted on 6 Feb 2020

HAL is a multi-disciplinary open access archive for the deposit and dissemination of scientific research documents, whether they are published or not. The documents may come from teaching and research institutions in France or abroad, or from public or private research centers.
L'archive ouverte pluridisciplinaire HAL, est destinée au dépôt et à la diffusion de documents scientifiques de niveau recherche, publiés ou non, émanant des établissements d'enseignement et de recherche français ou étrangers, des laboratoires publics ou privés. 


\title{
Determination of the melting curve of gold up to $110 \mathrm{GPa}$
}

\author{
Gunnar Weck,,${ }^{1, *}$ Vanina Recoules, ${ }^{1}$ Jean-Antoine Queyroux, ${ }^{1,2}$ Frédéric Datchi, ${ }^{2}$ Johann Bouchet, ${ }^{1}$ Sandra Ninet, ${ }^{2}$ \\ Gaston Garbarino, ${ }^{3}$ Mohamed Mezouar, ${ }^{3}$ and Paul Loubeyre ${ }^{1}$ \\ ${ }^{1}$ CEA, DAM, DIF, F-91297 Arpajon, France \\ ${ }^{2}$ Institut de Minéralogie, de Physique des Matériaux, et de Cosmochimie (IMPMC), Sorbonne Université, \\ CNRS UMR 7590, MNHN, 4 place Jussieu, F-75005 Paris, France \\ ${ }^{3}$ European Synchrotron Radiation Facility, BP 220, 38043 Grenoble, France
}

(Received 4 November 2019; revised manuscript received 17 December 2019; published 17 January 2020)

\begin{abstract}
The melting curve of gold has been measured up to $110 \mathrm{GPa}$ using laser-heated diamond anvil cells and synchrotron x-ray diffraction techniques. Accurate pyrometry temperature measurements and a homogeneous heating of the gold sample were achieved by implementing a sample assembly consisting of two boron-doped diamond cupped disks sandwiching the gold sample. In the investigated pressure range, the fcc solid gold remains stable up to melting. A clear structural signature of bulk melting is observed. Ab initio molecular dynamics simulations within the two-phase approach give a melting curve in good agreement with the experimental one. We discuss the validity of calculations based on the Lindemann criteria of melting which have been up to now used to obtain the melting line of $\mathrm{Au}$ in the $100 \mathrm{GPa}$ range.
\end{abstract}

DOI: 10.1103/PhysRevB.101.014106

\section{INTRODUCTION}

Gold is unique among metals for its exceptional chemical inertness, high ductility, optical properties, and structural stability up to extreme conditions of pressure and temperature. It is therefore a material of choice for many applications. In that context, many high-pressure studies have already been devoted to measuring the equation of state (EOS) of gold over an extended $P-T$ range $[1,2]$. The face-centered cubic (fcc) structure has been shown to remain stable up to at least $600 \mathrm{GPa}$ at ambient temperature [3]. Yet, there is evidence of a fcc-hcp transition above $240 \mathrm{GPa}$ under heating in the $1000 \mathrm{~K}$ range [4]. A formulation of the $P-V-T$ EOS (pressurevolume-temperature) of gold has been proposed, with parameters fitted to various measurements [5]. On the other hand, the determination of the melting curve remains poorly constrained by experiments. Measurements have been limited to $35 \mathrm{GPa}$ and experimental data above $15 \mathrm{GPa}$ are scattered. The melting line has been investigated by several authors in a large-volume apparatus up to 6-7 GPa, using the change of the sample electrical resistance to detect melting. The same criterium has also been used in recent experiments using the resistively heated diamond anvil cell (RHDAC) up to $21 \mathrm{GPa}$ [6]. The RHDAC was also used for an x-ray diffraction (XRD) experiment on gold where melting was obtained from the disappearance of the solid XRD signal [7]. To our knowledge there has only been one attempt to determine melting using laser heating in the diamond anvil cell (LHDAC) up to $35 \mathrm{GPa}$ [8]. These DAC studies report very different slopes of the melting lines above $15 \mathrm{GPa}$.

Over the past decade, great progress has been made in using the LHDAC to measure the melting line of metals.

*Corresponding author: gunnar.weck@cea.fr
Long-standing controversy in the determination of the melting line obtained either by dynamical compression or by the LHDAC has been resolved, as in the case of Ta [9] and Fe [10]. First, using the synchrotron XRD a bulk criterion of melting was implemented based on the appearance of the liquid diffuse scattering signal. Second, XRD measurements allow simultaneous detection of parasitic chemical reactions. Third, pyrometric measurements were validated from the measured thermal expansion of the metal. However, the LHDAC measurements on gold have remained challenging because (1) the high reflectivity of Au makes it difficult to absorb the IR laser radiation used for heating, and (2) the dispersion of the emissivity of gold in the visible invalidates the gray-body assumption that is used for pyrometric temperature measurements.

On the theoretical side, great progress has also been made in the calculation of melting lines using $a b$ initio calculations [11-15]. However, to our knowledge, no ab initio calculation of the melting curve of Au has been published so far. That is probably due to the computational time needed to treat the large number of electrons which have to be taken into account in the valence states. The melting line of $\mathrm{Au}$ was estimated using approaches based on classical molecular dynamics simulations with an embedded-atom method (EAM) potential [16] or the Lindemann approach [17] which apprehends the melting phenomena as a lattice instability [18-20].

Here, we show how accurate LHDAC measurements of the melting curve of Au can be made by encapsulating the $\mathrm{Au}$ sample in a B-doped diamond micro-oven inside the sample chamber. A homogeneous heating of the Au sample can be made and the temperature of Au is reliably estimated by pyrometry as the one of the heated diamond capsule. On the theoretical side, the ab initio molecular dynamics (AIMD) simulations within the two-phase approach (TPA), which directly compares the free energies of the solid and the liquid phases, are used to calculate the gold melting curve. 


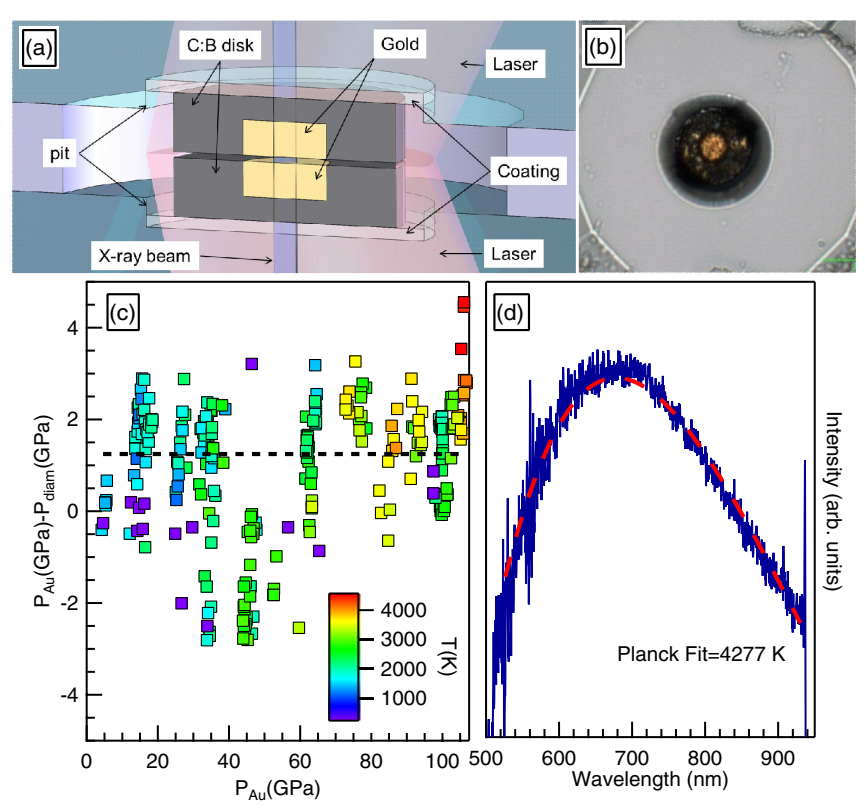

FIG. 1. Sample preparation and $P-T$ metrology. (a) Drawing of a cross-sectional view of the sample cavity located between the two diamond anvils of the DAC. Two cupped C:B disks are filled with gold and placed each in a pit (diameter: $45 \mu \mathrm{m}$; depth: $6 \mu \mathrm{m}$ ) realized by FIB at the center of the diamond anvil culet and coated with $\mathrm{LiF}$ or $\mathrm{Al}_{2} \mathrm{O}_{3}$. The two disks form a capsule heated from both sides by YLF laser beams. The laser focus is adjusted in order to fully illuminate the $\mathrm{C}: \mathrm{B}$ plates and reduce the radial temperature gradient. The $\mathrm{X}$-ray beam probes the gold sample confined between the two $\mathrm{C}: \mathrm{B}$ disks. Bottom: Determination of the thermodynamic conditions. (b) Difference between the pressures measured with the gold and diamond calibrants using the Dorogokupets semiempirical state equation [22]. (c) Thermal emission of a C:B disk at $104 \mathrm{GPa}$ and its analysis in the gray-body approximation. (d) The intensity vs wavelength spectrum fitted between $550 \mathrm{~nm}$ and $920 \mathrm{~nm}$ with a Planck distribution $\left(T_{\text {Planck }}=4277 \pm 3 \mathrm{~K}\right)$.

This paper is organized as followed. In Sec. II, the experimental methods and the data are presented. In Sec. III, the $a b$ initio calculations of the melting curve are described. The experimental and theoretical data are discussed in Sec. IV. Concluding remarks are presented in Sec. V.

\section{EXPERIMENTS}

\section{Methods}

The present experiments used membrane diamond anvil cells equipped with Boelher-Almax anvils of culet size ranging from 300 to $100 \mu \mathrm{m}$. Rhenium gaskets were used and the gasket hole was filled by the sample assembly and a pressuretransmitting medium (argon or $\mathrm{Al}_{2} \mathrm{O}_{3}$ ). A sketch of the sample assembly is shown in Fig. 1(a). It was formed by a 3-6 $\mu \mathrm{m}$ thick layer of gold sandwiched between two (opaque) borondoped diamond $(\mathrm{C}: \mathrm{B})$ cupped disk (30-35 $\mu \mathrm{m}$ in diameter, 5-10 $\mu \mathrm{m}$ thick) and loaded in the diamond anvil cell. The $\mathrm{C}: \mathrm{B}$ disks are focused ion beam (FIB) machined in the form of a cup in order to better confine the gold sample. In the first experimental run, a $30 \mu \mathrm{m}$ diameter disk and a $5 \mu \mathrm{m}$ thick gold foil were used for the sample assembly. In the following experiments, the samples were more easily prepared by coating a $2 \mu \mathrm{m}$ gold layer on the $\mathrm{C}$ :B disks directly. A pit was also FIB machined at the center of the diamond culets to ease the positioning of the disks and prevent their shift under load. A 1-2 $\mu \mathrm{m}$ thick $\mathrm{Al}_{2} \mathrm{O}_{3}$ or $\mathrm{LiF}$ layer was coated on the pitted anvil culets for thermal insulation. This geometry is similar to the one used for the determination of the nitrogen melting curve [21] and provides uniform heating of the sample.

The laser heating XRD experiments were performed at the ID27 beamline of the ESRF (see Ref. [23]). A monochromatic $\mathrm{x}$-ray beam of wavelength $\lambda=0.3738 \AA$ was focused to a $3 \mu \mathrm{m}$ FWHM spot size and the diffracted $\mathrm{x}$ rays were collected by a MarCCD detector. The sample-detector distance and x-ray beam position were calibrated with a $\mathrm{CeO}_{2}$ NIST standard powder. The typical exposure time was 2 to 8 seconds. The PyFAI software [24] as implemented in Dioptas [25] was used for integration of the XRD patterns. Double-sided laser heating was performed using 2 yttrium lithium fluoride (YLF) fiber lasers providing 10 to $100 \mathrm{~W}$ power with $0.1 \mathrm{~W}$ incremental resolution. The two laser heads were implemented on a vertical bench fixed to the sample translation stage. This allows us to realign the sample on the $\mathrm{x}$-ray beam at any time during heating without affecting the position of the laser-heated spot, thus improving the detection of a small amount of liquid, and thus the onset of melting. The lasers were focused to a $30-40 \mu \mathrm{m}$ spot size in order to homogeneously heat the $\mathrm{C}: \mathrm{B}$ disks.

The temperature was measured from both sides of the LHDAC. Chromatic-aberration-free reflective optics were used to collect the thermal emission pattern from a $3 \mu \mathrm{m}$ diameter area centered on the hot $\mathrm{C}: \mathrm{B}$ disks. A description of the pyrometric setup and its alignment using the X-ray induced sample fluorescence are given in Refs. [26,27]. The temperature was estimated by fitting the thermal emission spectrum with the Planck distribution in between 550 and $950 \mathrm{~nm}$ [see Fig. 1(c)]. The uncertainty was estimated from the dispersion of the two-color temperature [28] (which at $106 \mathrm{GPa}$ and $4277 \mathrm{~K}$ is found to be less than $\pm 200 \mathrm{~K}$ ).

Pressure was determined at ambient and at high temperature using the semiempirical equations of state of gold and of diamond, as constructed by Dorogokupets et al. [22]. Pressure increases of up to $10 \mathrm{GPa}$ could be observed between the ambient and the melting temperatures at the highest pressure. This large thermal pressure is due to the sample geometry where the C:B-gold assembly filled and thus heated almost the entire experimental cavity. Therefore, it was important to have a reliable in situ measure of the sample pressure at temperature. As shown in Fig. 1(b), the average difference between the pressures obtained with the gold and diamond gauges is less than $1.5 \mathrm{GPa}$ with a standard deviation of $2 \mathrm{GPa}$. After each heating cycle, the load was generally increased at high temperature in order to reduce the stresses in the sample chamber and preserve the integrity of the $\mathrm{C}$ : $\mathrm{B}$ disks.

Melting points were determined along heating cycles. The XRD patterns of the sample were collected while temperature was stepwise increased and decreased. No chemical reaction was detected between gold, argon, diamond, and the $\mathrm{Al}_{2} \mathrm{O}_{3}$ layer, which altogether allow indexing of all the diffraction peaks observed on each image plate. Melting was associated 


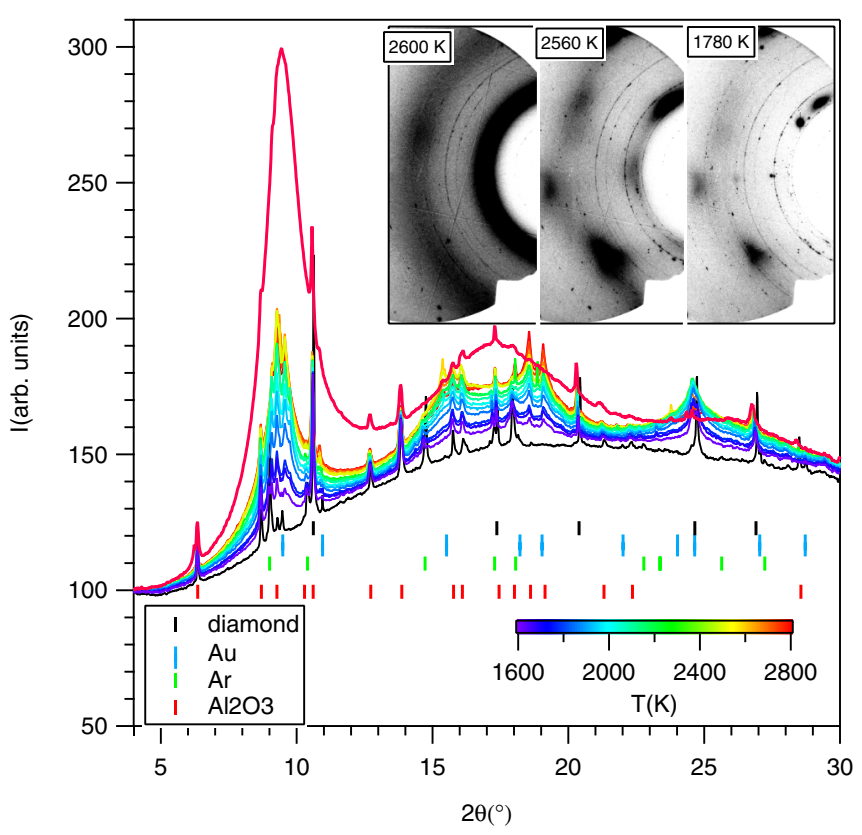

FIG. 2. X-ray diffraction patterns collected at $36.5 \mathrm{GPa}$, between 2600 and $1600 \mathrm{~K}$. The spectrum in red, collected at $2600 \mathrm{~K}$ (above melting), shows the first three diffuse rings of the liquid gold structure factor. Below melting, the TDS from the hot solid is composed of a background signal added to the Compton contribution from the anvils, and a broad, temperature-dependent footprint around each Bragg peak. The spectrum in black was obtained after quenching the sample at $300 \mathrm{~K}$. The sample pressure dropped at $27 \mathrm{GPa}$. Gold, diamond, and $\mathrm{Al}_{2} \mathrm{O}_{3}$ peaks are labeled. The saturated $\mathrm{Au}$ single-crystal peaks which formed under heating have been masked before integrating the image plate, which explains why the $\mathrm{Au}$ diffraction peaks appear relatively weak on the spectra. Inset: X-ray diffraction images collected at three different temperatures for a $\mathrm{C}$ :B-gold assembly in argon at $P=36.5 \mathrm{GPa}$. At $2600 \mathrm{~K}$, gold is fully melted and the first two liquid diffuse scattering rings are observed. At $2560 \mathrm{~K}$ and $1780 \mathrm{~K}$, gold is solid and the temperature diffuse scattering around the gold XRD spots is observed.

with the appearance of broad diffuse x-ray scattering rings from the liquid sample fraction.

At pressures below $50 \mathrm{GPa}$, these diffuse rings were intense, directly visible on the diffraction images (see inset of Fig. 2), and abruptly appeared (disappeared) when crossing up (down) the melting temperature. This indicates that most, if not all, of the gold sample melted at the transition and that temperature gradients were small inside the volume enclosed by the cupped $\mathrm{C}: \mathrm{B}$ disks. Below the melting temperature, the gold single-crystal diffraction peaks and a temperature diffuse scattering (TDS) contribution appear simultaneously with the disappearance of the liquid signal. The TDS both broadens the base of the Bragg diffraction peaks, clearly visible on the integrated diffraction patterns, and gives a smooth background signal that adds to the Compton contribution from the diamond anvils. A series of patterns collected below melting and just above are compared in Fig. 2. This shows that the solid TDS contribution and the liquid diffuse scattering are clearly distinct.

Below $50 \mathrm{GPa}$, a molten state of gold was stabilized inside the $\mathrm{C}: \mathrm{B}$ capsule. By adjusting the power of the two YLF

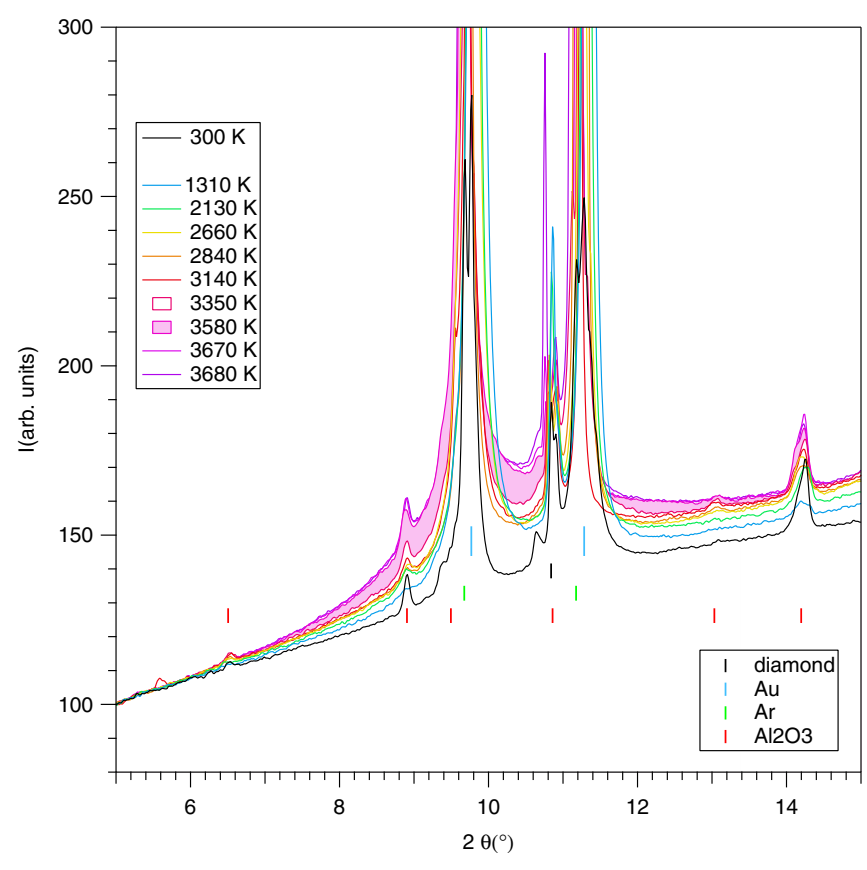

FIG. 3. X-ray diffraction patterns collected at $78 \mathrm{GPa}$ during a heating cycle. Each spectrum is plotted with a different color and labeled with the temperature. A reference spectrum collected after quench is plotted in black showing the ambient temperature sample diffraction peaks and the diamond anvil Compton scattering. The TDS contribution from the hot gold solid appears as a smooth signal added to the Compton contribution from the anvils. The liquid signal appears at $3580 \mathrm{~K}$ as a weak broad diffuse ring centered at $10.5^{\circ}$. The sample pressure dropped at $67 \mathrm{GPa}$ after quench. Gold, diamond, and $\mathrm{Al}_{2} \mathrm{O}_{3}$ peak positions are indicated by vertical tick marks.

lasers to balance heating on both sides of the sample, an accurate determination of the melting temperature was obtained. Above $50 \mathrm{GPa}$, gold could only be partially melted and the liquid signal appeared as a weak diffuse ring (see Fig. 3) that adds to the Compton diamond background and the diffraction peaks from the remaining gold solid sample. This contribution while weak still appeared abruptly at melting. The liquid signal was slightly growing with temperature but the solid diffraction peaks remained intense $500 \mathrm{~K}$ above melting, indicating an important temperature gradient in the sample. In addition, melting was often rapidly followed by a drop of the overall sample signal most probably due to a partial leak of the gold sample outside the C:B capsules. To overcome this problem, the sample XRD images and the temperature were continuously collected while ramping up the laser power with a second timescale resolution. This method leads to larger uncertainties on the melting temperature determination.

The present Au melting data set is plotted in Fig. 4. Seventeen melting data points have been collected in between 5 and $110 \mathrm{GPa}$ by studying a total of ten samples and are presented in Table I. The fcc structure of solid gold is found stable over the entire $P-T$ range covered. The melting data points are well fitted by a Simon-Glatzel equation [29] of the form $T_{m}=T_{0}\left(1+P_{m} / a\right)^{b}$ where $T_{0}=1337 \mathrm{~K}$ is the melting temperature at ambient pressure, $a=22.265 \pm 1.83 \mathrm{GPa}$, and $b=0.662 \pm 0.03$. 


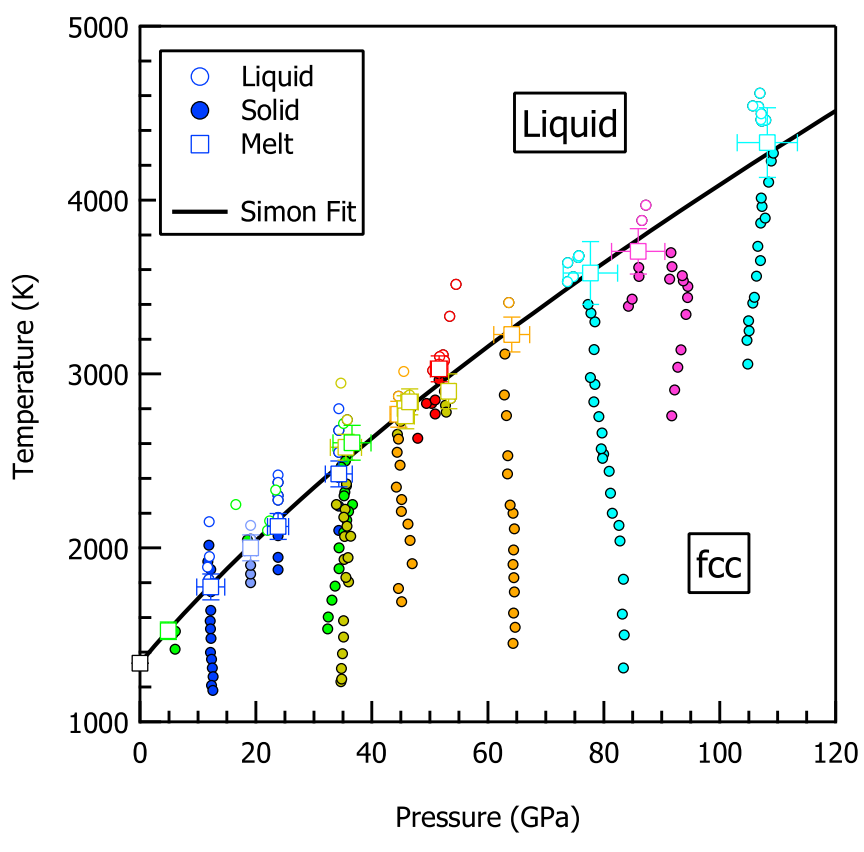

FIG. 4. Gold melting curve measured in this study by XRD. The pressure and temperature conditions at which XRD patterns have been collected are shown. The empty (plain) circles represent XRD patterns with liquid (solid only) gold signal. Each color represents a different sample. Pressure and temperature error bars are shown for the melting points only (represented by empty squares). The black line is a fit of the Simon-Glatzel equation to the melting points: $T_{m}=T_{0}\left(1+P_{m} / a\right)^{b}$ with $T_{0}=1337 \mathrm{~K}, a=22.265 \pm 1.83 \mathrm{GPa}$, and $b=0.662 \pm 0.03$.

TABLE I. Experimental melting points of gold determined in this work with associated error bars ( $T_{\text {melt }}$ in $\mathrm{K}$ and $P_{\text {melt }}$ in GPa). The error in pressure is given by the difference between the pressures measured with the gold and diamond calibrants. The error in temperature is given by the dispersion of the two-color pyrometry.

\begin{tabular}{lccc}
\hline \hline$P_{\text {melt }}$ & $T_{\text {melt }}$ & $\pm \Delta P_{\text {melt }}$ & $\pm \Delta T_{\text {melt }}$ \\
\hline 4.9 & 1523 & 0.5 & 50 \\
12.2 & 1776 & 2.4 & 75 \\
19.1 & 2000 & 1.0 & 75 \\
23.8 & 2124 & 1.8 & 75 \\
34.3 & 2426 & 2.3 & 75 \\
35.5 & 2579 & 2.7 & 75 \\
36.6 & 2605 & 3.3 & 100 \\
44.2 & 2768 & 1.5 & 75 \\
45.3 & 2760 & 1.5 & 75 \\
45.7 & 2800 & 1.4 & 75 \\
46 & 2840 & 1.5 & 75 \\
51.6 & 3029 & 1.5 & 75 \\
52.6 & 2900 & 1 & 100 \\
63.3 & 3227 & 3.1 & 100 \\
75.5 & 3580 & 4.7 & 180 \\
85.9 & 3705 & 4.6 & 130 \\
106 & 4330 & 5.2 & 200 \\
\hline \hline
\end{tabular}

\section{CALCUlations}

To calculate the melting temperature with ab initio methods we use the two-phase approach [30,31] (TPA). Simulations were performed on supercells containing the two phases, solid and liquid, separated by an interface. This is different from the coexistence method where the liquid and the solid are also placed in the same computational cell but simulations are performed in the $N V E$ ensemble. Compared to the "heat until it melts" (HUM) method where the solid phase is heated gradually until melting occurs, the TPA avoids the superheating effect due to the homogeneous heating [14]. The TPA has been widely used and is known to give excellent comparison with experiments (see for example Al [14], Fe [13,32], $\mathrm{LiH}$ [31], Ti [15], or $\mathrm{Ni}$ [33]). The counterpart is the increased computational cost due to the doubling of the supercell that may become critical for high- $Z$ elements like gold. We used supercells of 216 atoms corresponding to $6 \times 3 \times 3$ cubic fcc cells of 4 atoms, a supercell size similar to the ones used for transition metals $\mathrm{Ti}$ [15] or Fe [32]. The simulations were performed for 8 densities: 18.3, 19.3, 20.2, 20.70, 21.15, 22, 23 , and $24 \mathrm{~g} / \mathrm{cm}^{3}$. The two-phase simulations were prepared by starting from the perfect fcc crystal and by thermalizing at a temperature below melting for $1 \mathrm{ps}$. Then half of the atoms were kept fixed at their positions while the other half were melted using a high-temperature value. Once the positions for the solid and the liquid part were obtained, several $N V T$ molecular dynamics simulations were performed for several temperatures with an increment of $200 \mathrm{~K}$. Only one phase was preserved at the end of the simulation: above $T_{m}$, the solid part becomes liquid, whereas below $T_{m}$, the liquid part crystallizes. If the energy and volume are appropriately chosen, the liquid and the solid could coexist for a long time but it has been shown that this method requires larger supercells and a longer computational time [13].

Our simulations were performed using the ABINIT package $[34,35]$ in the framework of density functional theory (DFT) using the projector augmented wave (PAW) method $[36,37]$ and by means of the local density approximation (LDA) [38]. The LDA functional was preferred over the generalized gradient approximation (GGA) since for $\mathrm{Au}$ it gives better results with respect to the experimental $300 \mathrm{~K}$ isotherm [39]. We used a PAW atomic data set generated using ATOMPAW [40,41] that has been extensively tested $[39,42]$. The PAW radius $r_{\text {PAW }}$ equals $1.32 \AA$, with $5 d$ and $6 s$ states as valence electrons (11 electrons). The cutoff energy chosen for the plane wave set along the simulations is $408 \mathrm{eV}$. We have validated our PAW atomic data set for this study against the XRD data of Takemura and Dewaele [2], and the semiempirical Vinet equation of state (EOS) proposed by Dorogokupets and Oganov [22] used to determine the pressure in our experiments; see Sec. II. We show in Fig. 5 the pressure obtained at $300 \mathrm{~K}$ with two sets of AIMD calculations. The first set is performed with the supercell used in our TPA calculations and the $\Gamma$ point only, and the second one with a $4 \times 4 \times 4$ cubic fcc supercell containing 256 atoms and a $2 \times 2 \times 2$ k-point grid. The pressure obtained with the larger supercell is close to the experimental data and the semiempirical EOS, with a small underestimation of the pressure at low densities and a decrease of this discrepancy with pressure. This is in 


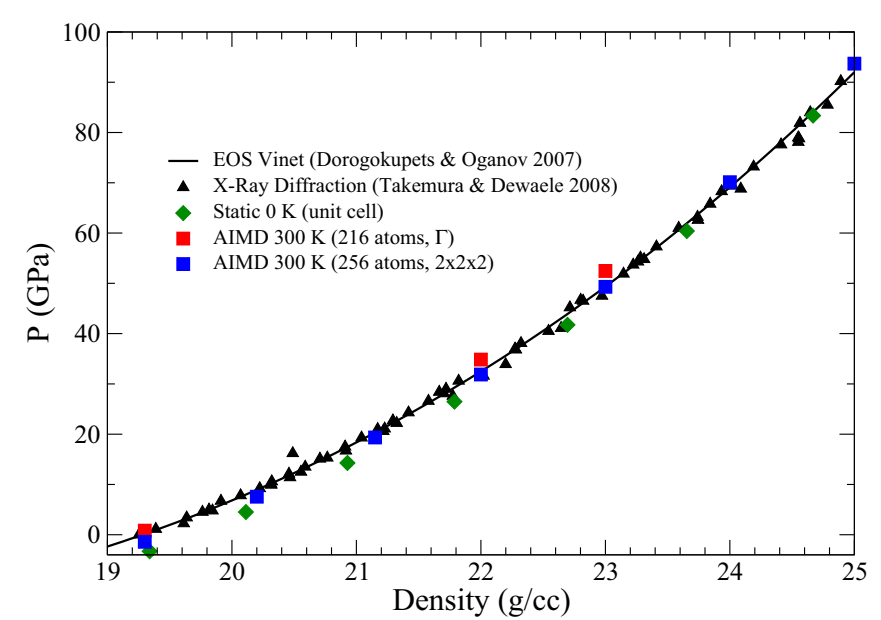

FIG. 5. Isothermal equation of state of gold calculated by $a b$ initio DFT methods in the present work. Green lozenge: 0 K EOS; blue square: $300 \mathrm{~K}$ EOS calculated with a supercell of 256 atoms and a $2 \times 2 \times 2 \mathbf{k}$-point grid, and (red square) with the supercell used in the TPA, 216 atoms and the $\Gamma$ point. The black line is the semiempirical Vinet EOS proposed by Dorogokupets and Oganov [22], and the black triangle the XRD data obtained by Takemura and Dewaele at $300 \mathrm{~K}$ [2].

agreement with the comparison between XRD data and PAW calculations done by Dewaele et al. [39]. In the latter work, the calculations were performed at $0 \mathrm{~K}$ with the fcc unit cell, and the thermal pressure was estimated using a Debye model, whereas the thermal pressure is here directly estimated in our AIMD simulations. The simulations with the TPA supercell slightly overestimated the pressure when compared to the 256 atoms supercell (between 1.5 GPa and $3 \mathrm{GPa}$ ). This is due to a coarser k-point grid ( $\Gamma$ point only versus $2 \times 2 \times 2$ grid)

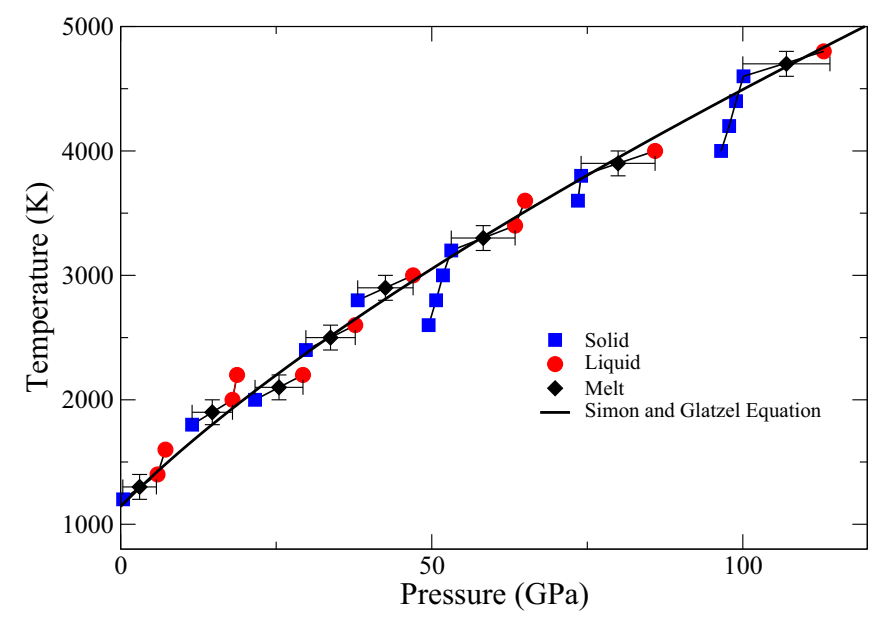

FIG. 6. Ab initio melting curve of Au obtained with the TPA. The blue square represents $(P, T)$ points for which the liquid part crystallizes while the red circle is $(P, T)$ points for which the solid part melts. The melting temperatures for each isochore (black diamonds) are between the solid with the highest temperature and the liquid with the lowest one. The black line is a fit of the Simon-Glatzel equation to the melting points $\left[T_{m}=T_{0}\left(1+P_{m} / a\right)^{b}\right.$ with $T_{0}=1181 \mathrm{~K}, a=$ $17.94 \mathrm{GPa}$, and $b=0.09]$.
TABLE II. Theoretical melting points of gold determined in this work with associated error bars $\left(T_{\text {melt }}\right.$ and $\Delta T_{\text {melt }}$ in $\mathrm{K}$ and $P_{\text {melt }}$ and $\Delta P_{\text {melt }}$ in $\mathrm{GPa}$ ).

\begin{tabular}{lccc}
\hline \hline$P_{\text {melt }}$ & $T_{\text {melt }}$ & $\pm \Delta P_{\text {melt }}$ & $\pm \Delta T_{\text {melt }}$ \\
\hline 3.05 & 1300 & 3 & 100 \\
14.7 & 1900 & 3 & 100 \\
25.4 & 2100 & 4 & 100 \\
34.7 & 2500 & 3 & 100 \\
42.6 & 2900 & 4 & 100 \\
58.2 & 3300 & 5 & 100 \\
80.0 & 3900 & 6 & 100 \\
107.0 & 4671 & 7 & 100 \\
\hline \hline
\end{tabular}

used with the TPA to reduce the computational time. We will discuss this effect in Sec. IV.

In Fig. 6, the Au melting temperatures obtained using the TPA approach along 8 isochores are presented. The final states of the supercell, either solid or liquid, are also plotted. The melting data point is determined as the pressure and temperature averages between the hottest solid point and the coldest liquid point. Note that some simulations at conditions far from melting points were stopped before reaching equilibration and are therefore not plotted in Fig. 6. Table II gives the computed values. The error in melting temperature is given by the temperature steps we used. The error in pressure is given by the pressure difference between the solid and the liquid phase.

The fit of the $a b$ initio melting points with the SimonGlatzel equation gives $T_{0}=1181 \mathrm{~K}$, the melting temperature at ambient pressure, $a=17.94 \mathrm{GPa}$, and $b=0.709$.

\section{DISCUSSION}

\section{A. Comparison to previous experiments}

In Fig. 7, the present Au melting data points are compared to previous determinations. Many experiments were conducted using large-volume high-pressure apparatuses up to a maximum pressure of $7 \mathrm{GPa}$. The signature of melting was then evidenced either by differential thermal measurements or electrical measurements (EMs). These studies were recently collected and reviewed [43] showing some dispersion of the various data (about $60 \mathrm{~K}$ at $7 \mathrm{GPa}$ with $T_{\text {melt }}=1600 \mathrm{~K}$ ). The present data points are in good agreement with this low-pressure data set, being slightly nearer to the EM melting points, with a maximum discrepancy of $40 \mathrm{~K}$ and $20 \mathrm{~K}$ at $6 \mathrm{GPa}$, respectively from the work of Errandonea [44] and of Mitra et al. [45]. Melting of gold was also measured in a resistive heated diamond anvil cell (RHDAC) experiment using XRD (up to $9 \mathrm{GPa}$ ), or electrical measurements (e-RHDAC, up to $21 \mathrm{GPa}$ ) and in a laser-heated diamond anvil cell experiment (up to $35 \mathrm{GPa}$ ) using as melting criteria the visual observation of macroscopic changes of the sample shape into small round balls (o-LHDAC) [8]. These various DAC experiments agree within the error bars below $15 \mathrm{GPa}$, but the last e-RHDAC melting point obtained at $21 \mathrm{GPa}$ underestimates the melting temperature by $200 \mathrm{~K}$ and suggests a very different slope for the melting curve above this pressure. Also should be noted the good agreement (to 


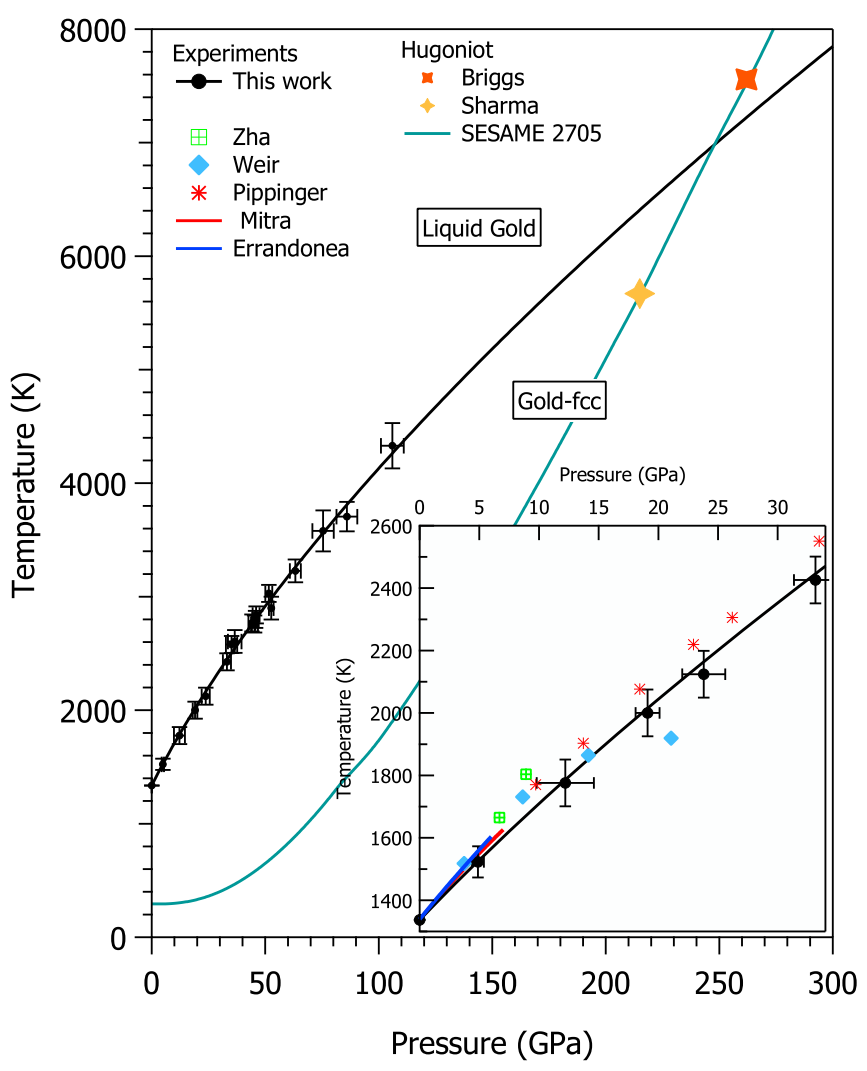

FIG. 7. Melting curve of gold obtained in this work from XRD measurements. Previous determinations from experiments (labeled Zha [7], Weir [6], Pippinger [8], Mitra [45], Errandonea [44]) are displayed in the figure inset. The plain green line shows the Hugoniot of gold calculated with the SESAME 2008 table [5].

within $100 \mathrm{~K}$ ) between our $\mathrm{x}$-LHDAC and the o-LHDAC measurements. This could be fortuitous since the same oLHDAC approach applied to determine the iron melting curve underestimated the melting temperature by at least $300 \mathrm{~K}$ at $43 \mathrm{GPa}$ [8]. Moreover pyrometric measurements performed on heated gold are not reliable due to the breakdown of the gray-body approximation.

Melting of gold was also investigated by dynamical compression. The gold Hugoniot data have been collected up to $580 \mathrm{GPa}$ using a single- [46] or double-stage gas gun [47]. No sign of melting could be detected from the macroscopic observables, shock and particle velocities. This was ascribed to the small-volume discontinuity on the melting curve at very high pressure [18]. Recently, two independent laser shock compression experiments on $\mathrm{Au}$, coupled to synchrotron XRD, were conducted to probe the structural evolution along the Hugoniot $[48,49]$. The melting could be detected by the appearance of a diffuse scattering signal from the liquid part of the sample. Both experiments suggest a triple point, where the fcc solid, the body-centered cubic (bcc) solid, and the fluid cross in the Au phase diagram, located near the principal Hugoniot. However, the reported transition pressures by the two studies differ by more than $40 \mathrm{GPa}$, from 176 to $223 \mathrm{GPa}$ and from 220 to $262 \mathrm{GPa}$ for the fcc-bcc and the melting transitions, respectively. Since the two studies were performed under the same experimental conditions and the reported XRD

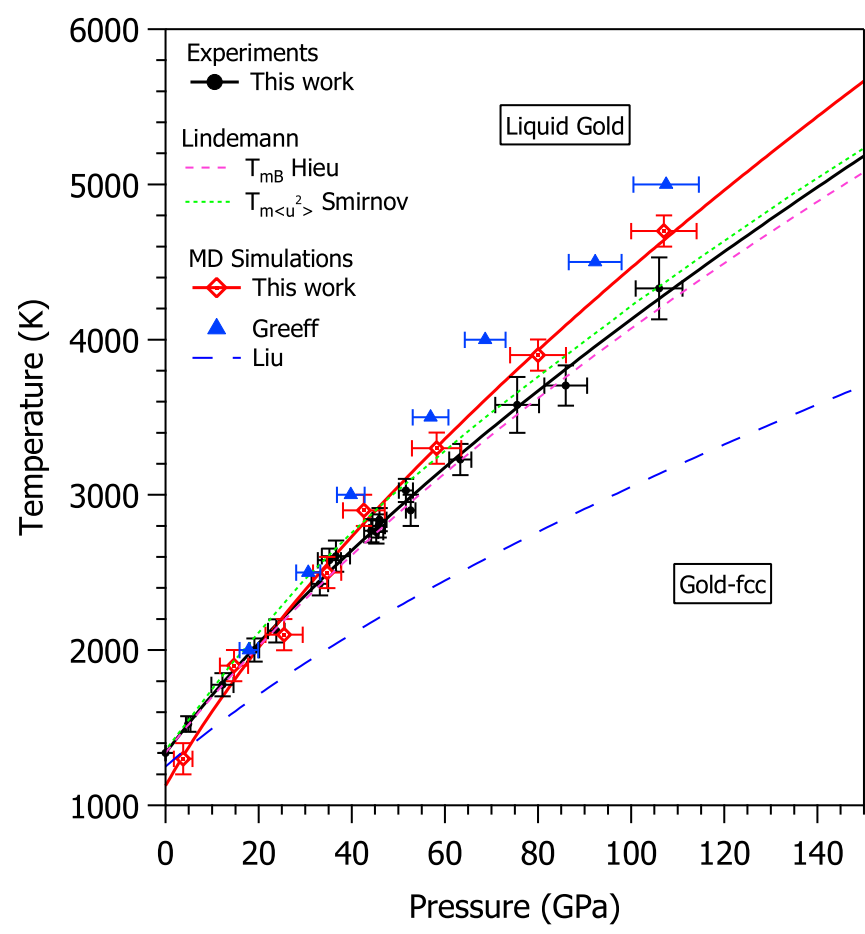

FIG. 8. Melting curves of gold obtained in this work from XRD measurements and $a b$ initio simulations. Previous determinations from $a b$ initio simulations (labeled Greeff [18]) and classical molecular dynamics simulations (labeled Liu [52]) are shown for comparison. The green and red dashed lines correspond to the melting curves determined using the Lindemann criterion calculated respectively from the atomic mean-squared displacements $\left(T_{m\langle u\rangle^{2}}\right)$ obtained by $a b$ initio simulations [20] or from the bulk modulus $\left(T_{\mathrm{mB}}\right)$ [19] determined by XRD [39].

patterns are very similar, this illustrates the difficulty in precisely positioning the melting point in shocked Au even with access to the atomistic arrangement in the shocked Au sample. This essentially stems from the gradual nature of melting along the Hugoniot which extends over $80 \mathrm{GPa}$. Hence the onset on melting needs to be detected from a very small molten fraction of the sample. The change of the melting slope due to the existence of the fcc-bcc-liquid triple point is expected to be small since the structural data imply a small-volume change at the fcc-bcc transition [49]. As shown in Fig. 7, the Hugoniot of gold [5] should cross the extrapolated Simon-Glatzel fit of the Au melting curve at $248 \mathrm{GPa}$ and $7000 \mathrm{~K}$. This is in better agreement with the highest value reported in the dynamical experiments [49]. However comparison between static and dynamic compression data should be made with caution since the temperature is only estimated in shock wave experiments.

\section{B. Comparison to simulations}

Various calculations of the melting line of gold are compared to the present experimental determination in Fig. 8. In Ref. [20], an empirical evaluation of the gold melting curve was proposed using the Lindemann criterion, which stipulates that melting occurs when the atomic mean-squared displacement reaches a critical fraction (the Lindemann constant) of the interatomic distance. The Lindemann constant 
was chosen to reproduce the melting temperature of gold at ambient pressure. Mean-squared displacements were evaluated from the phonon density of states computed by ab initio simulations. As shown in Fig. 8, the good agreement with our experimental measurements confirms the robustness of the Lindemann criteria for simple metals. Using a generalization of the Lindemann law [50], Hieu et al. [19] proposed an analytical expression for the volume dependence of the melting temperature based on Grüneisen parameters. Several formulations were proposed for the pressure dependence of Grüneisen parameters, obtained either from first-principles calculations or from the bulk modulus or Debye-Waller factor measurements [51]. Interestingly, the parameters that are derived from the bulk modulus (using the equation of state measured in the DAC by Dewaele [39]) give the best agreement with our experimental data (see Fig. 8).

In Ref. [18], gold melting points were obtained by comparing the free energies of the solid and the liquid using "empirical model assumptions for the liquid" and the quasiharmonic approximation (QHA) for the solid. The electronic structure calculations were performed within the LDA as in this work. As shown in Fig. 8, the melting temperature is significantly overestimated with respect to the experiment probably due to the too simplistic description of the fluid phase and/or anharmonic effects in the solid beyond the QHA. Moreover, melting along the gold Hugoniot was predicted to occur at $280 \mathrm{GPa}$ with a significant pressure jump of $30 \mathrm{GPa}$ (due to a calculated large-volume discontinuity at melting), which was not experimentally observed in subsequent shock measurements performed up to $580 \mathrm{GPa}$ [53].

Classical molecular dynamics simulations have been performed on gold in order to compare two melting simulation methods [52]: the shock melting (SM) method and the TPA. The EAM potential was used. This potential [16] was fitted to several experimental data: the equilibrium lattice constant, the sublimation energy, the bulk modulus, the elastic constants, and the vacancy formation energy. It is important to mention that this potential was not originally designed to calculate a melting curve, especially at high pressure, but other physical properties such as the activation energies for self-diffusion [16]. The transferability of this potential to high temperature $(1000 \mathrm{~K})$ at ambient pressure was recently tested [54]. Melting simulations were performed on a supercell containing 20736 atoms to ensure convergence of the melting temperature while the SM method was tested on a much smaller number of atoms (640 atoms) to test the reliability of the method. The two calculations give the same melting curve which is much lower than experiment (by $87 \mathrm{~K}$ at ambient pressure and up to $1000 \mathrm{~K}$ at $100 \mathrm{GPa}$; see Fig. 8). This raises the question of the ability of classical simulations to accurately reproduce (or predict) melting curves with empirical potentials. Further simulations should be performed with potentials that better reproduce the melting temperature of gold at ambient pressure or which have been fitted to high-temperature data to test their transferability.

To circumvent the issue of transferability inherent to the classical molecular dynamics, we use DFT to calculate the interatomic forces. The cost for better accuracy is the computational time of AIMD. Therefore these simulations can only be performed on smaller supercells compared to the classical ones (basically, hundreds of atoms compared to thousands). Overall, our TPA results are in good agreement with our experimental data; see Fig. 8. At low pressure $(\sim 3 \mathrm{GPa})$ TPA gives a melting temperature slightly lower than the experimental one $(1300 \pm 100 \mathrm{~K}$ compared to $1500 \mathrm{~K})$ while at high pressure the calculated melting temperature is above the experimental one (around $370 \mathrm{~K}$ difference at $107 \mathrm{GPa}$ ). If we take into account the small overestimation of the pressure due to the k-point sampling, see Sec. III, this improves the comparison at low pressure but deteriorates it at higher pressure. The discrepancy between simulations with 216 atoms and $\Gamma$-point sampling and those with 256 atoms with $2 \times 2 \times 2 \mathbf{k}$-point grid sampling is less than $3 \mathrm{GPa}$ for the highest pressure, which is below the error bar obtained for the melting pressure. We believe that the main source of error in our calculations comes from the size of the simulation supercell. Unfortunately, the calculations with a larger supercell $(8 \times 4 \times 4$ fcc cubic cell, 512 atoms $)$ are too costly in the case of Au, but the comparison has been made for a simpler metal, Al. In that work, Bouchet et al. [14] compared the melting curve of Al obtained with the TPA and fcc supercells with 108, 216, and 512 atoms. They observed a slow convergence of the melting temperature with the supercell size with a difference around $10 \%$ in the melting temperature between 216 and 512 atoms. Moreover the discrepancies increase with pressure, which may explain why we find a better agreement at low pressure with the experimental data. Note that the use of GGA instead of LDA could also improve the comparison at high pressure with a lowering of the melting temperature as is the case for example in $\mathrm{MgO}$ [12], but the comparison would have been worse at low pressure.

\section{CONCLUSION}

In conclusion, we have measured the melting curve of gold to above $100 \mathrm{GPa}$ with high accuracy by combining in situ measurements of the sample pressure and temperature with XRD-based melting criteria. The gold $P V T$ data points are consistent with the semiempirical EOS of Dorogokupets [22]. The melting curve of gold was compared to some estimations based on the Lindemann criterion. Our data can be well reproduced with a modified Lindemann formula using a volume-dependent Grüneisen parameter determined from the volume derivative of the bulk modulus. The latter can be obtained from XRD measurements of the equation of state in the DAC at ambient temperature. This highlights a strong correlation between the EOS and the thermal stability for a simple metal. Finally, the extended melting data set shows that the EAM potential [16] cannot reproduce the melting curve of gold at high pressure. By contrast, AIMD simulations, using the same method (the two-phase approach) but a smaller supercell, are in better agreement with experiment.

\section{ACKNOWLEDGMENTS}

We acknowledge the European Synchrotron Radiation Facility for provision of beam time under the auspices of the long-term project HD-463 and run HC2490, and financial support from the Agence Nationale de la Recherche under Grant No. ANR-13-BS04-0015 (MOFLEX). 
[1] D. Batani, A. Balducci, D. Beretta, A. Bernardinello, T. Löwer, M. Koenig, A. Benuzzi, B. Faral, and T. Hall, Phys. Rev. B 61, 9287 (2000).

[2] K. Takemura and A. Dewaele, Phys. Rev. B 78, 104119 (2008).

[3] A. Dewaele, P. Loubeyre, F. Occelli, O. Marie, and M. Mezouar, Nat. Commun. 9, 2913 (2018).

[4] L. Dubrovinsky, N. Dubrovinskaia, W. A. Crichton, A. S. Mikhaylushkin, S. I. Simak, I. A. Abrikosov, J. S. de Almeida, R. Ahuja, W. Luo, and B. Johansson, Phys. Rev. Lett. 98, 045503 (2007).

[5] J. Boettger, K. G. Honnell, J. H. Peterson, C. Greeff, and S. Crockett, in Proceedings of the Conference of the American Physical Society Topical Group on Shock Compression of Condensed Matter 2011, edited by M. L. Elert, W. T. Buttler, J. P. Borg, J. L. Jordan, and T. J. Vogler, AIP Conf. Proc. No. 1426 (AIP, New York, 2012), p. 812.

[6] S. T. Weir, D. D. Jackson, S. Falabella, G. Samudrala, and Y. K. Vohra, Rev. Sci. Instrum. 80, 013905 (2009).

[7] C.-S. Zha and W. A. Bassett, Rev. Sci. Instrum. 74, 1255 (2003).

[8] T. Pippinger, L. Dubrovinsky, K. Glazyrin, R. Miletich, and N. Dubrovinskaia, Física de la Tierra 23, 29 (2011).

[9] A. Dewaele, M. Mezouar, N. Guignot, and P. Loubeyre, Phys. Rev. Lett. 104, 255701 (2010).

[10] S. Anzellini, A. Dewaele, M. Mezouar, P. Loubeyre, and G. Morard, Science 340, 464 (2013).

[11] D. Alfè, G. D. Price, and M. J. Gillan, Phys. Rev. B 64, 045123 (2001)

[12] D. Alfè, Phys. Rev. Lett 94, 235701 (2005).

[13] D. Alfè, Phys. Rev. B 79, 060101(R) (2009).

[14] J. Bouchet, F. Bottin, G. Jomard, and G. Zérah, Phys. Rev. B 80, 094102 (2009).

[15] V. Stutzmann, A. Dewaele, J. Bouchet, F. Bottin, and M. Mezouar, Phys. Rev. B 92, 224110 (2015).

[16] J. B. Adams, S. M. Foiles, and W. G. Wolfer, J. Mater. Res. 4, 102 (1989).

[17] F. A. Lindemann, Physik. Z. 11, 609 (1910).

[18] C. W. Greeff and M. J. Graf, Phys. Rev. B 69, 054107 (2004).

[19] H. K. Hieu and N. N. Ha, AIP Adv. 3, 112125 (2013).

[20] N. A. Smirnov, Y. M. Chen, X. R. Chen, and Q. Wu, J. Phys.: Condens. Matter 29, 105402 (2017).

[21] G. Weck, F. Datchi, G. Garbarino, S. Ninet, J. A. Queyroux, T. Plisson, M. Mezouar, and P. Loubeyre, Phys. Rev. Lett. 119, 235701 (2017).

[22] P. I. Dorogokupets and A. R. Oganov, Phys. Rev. B 75, 024115 (2007).

[23] M. Mezouar, W. A. Crichton, S. Bauchau, F. Thurel, H. Witsch, F. Torrecillas, G. Blattmann, P. Marion, Y. Dabin, J. Chavanne et al., J. Synch. Radiat. 12, 659 (2005).

[24] G. Ashiotis, A. Deschildre, Z. Nawaz, J. P. Wright, D. Karkoulis, F. E. Picca, and J. Kieffer, J. Appl. Cryst 48, 510 (2015).

[25] C. Prescher and V. B. Prakapenka, High Pressure Res. 35, 223 (2015).

[26] S. Petitgirard, A. Salamat, P. Beck, G. Weck, and P. Bouvier, J. Synch. Radiat. 21, 89 (2014).

[27] A. Dewaele, M. Mezouar, N. Guignot, and P. Loubeyre, Phys. Rev. B 76, 144106 (2007).

[28] L. R. Benedetti and P. Loubeyre, High Pressure Res. 24, 423 (2004).
[29] F. Simon and G. Glatzel, Z. Anorg. Allg. Chem. 178, 309 (1929).

[30] A. B. Belonoshko, Geochim. Cosmochim. Acta 58, 4039 (1994).

[31] T. Ogitsu, E. Schwegler, F. Gygi, and G. Galli, Phys. Rev. Lett 91, 175502 (2003).

[32] J. Bouchet, S. Mazevet, G. Morard, F. Guyot, and R. Musella, Phys. Rev. B 87, 094102 (2013).

[33] M. Pozzo and D. Alfè, Phys. Rev. B 88, 024111 (2013).

[34] The ABINIT code is a common project of the Catholic University of Louvain (Belgium), Corning Incorporated, CEA (France), and other collaborators, http://www.abinit.org.

[35] X. Gonze, B. Amadon, P.-M. Anglade, J.-M. Beuken, F. Bottin, P. Boulanger, F. Bruneval, D. Caliste, R. Caracas, M. Côté et al., Comput. Phys. Commun. 180, 2582 (2009).

[36] P. E. Blöchl, Phys. Rev. B 50, 17953 (1994).

[37] M. Torrent, F. Jollet, F. Bottin, G. Zerah, and X. Gonze, Comput. Mater. Sci. 42, 337 (2008).

[38] D. M. Ceperley and B. J. Alder, Phys. Rev. Lett. 45, 566 (1980).

[39] A. Dewaele, M. Torrent, P. Loubeyre, and M. Mezouar, Phys. Rev. B 78, 104102 (2008).

[40] N. Holzwarth, A. Tackett, and G. Matthews, Comput. Phys. Commun. 135, 329 (2001).

[41] ATOMPAW is a general license public code developed at Wake Forest University. Some of its capabilities have been developed at the Commissariat à l'Énergie Atomique, http://pwpaw.wfu. edu.

[42] B. Holst, V. Recoules, S. Mazevet, M. Torrent, A. Ng, Z. Chen, S. E. Kirkwood, V. Sametoglu, M. Reid, and Y. Y. Tsui, Phys. Rev. B 90, 035121 (2014).

[43] M. Berrada, R. A. Secco, and W. Yong, High Pressure Res. 38, 367 (2018).

[44] D. Errandonea, J. Appl. Phys. 108, 033517 (2010).

[45] N. R. Mitra, D. L. Decker, and H. B. Vanfleet, Phys. Rev. 161, 613 (1967).

[46] S. Marsh (ed.), LASL Shock Hugoniot Data (University of California Press, Berkeley, 1980).

[47] M. Yokoo, N. Kawai, K. G. Nakamura, K. I. Kondo, Y. Tange, and T. Tsuchiya, Phys. Rev. B 80, 104114 (2009).

[48] S. M. Sharma, S. J. Turneaure, J. M. Winey, Y. Li, P. Rigg, A. Schuman, N. Sinclair, Y. Toyoda, X. Wang, N. Weir et al., Phys. Rev. Lett. 123, 045702 (2019).

[49] R. Briggs, F. Coppari, M. G. Gorman, R. F. Smith, S. J. Tracy, A. L. Coleman, A. Fernandez-Pañella, M. Millot, J. H. Eggert, and D. E. Fratanduono, Phys. Rev. Lett. 123, 045701 (2019).

[50] Y. Wang, R. Ahuja, and B. Johansson, Phys. Rev. B 65, 014104 (2001).

[51] M. J. Graf, C. W. Greeff, and J. C. Boettger, in Proceedings of the Conference of the American Physical Society Topical Group on Shock Compression of Condensed Matter, edited by M. D. Furnish, Y. M. Gupta, and J. W. Forbes, AIP Conf. Proc. No. 706 (AIP, New York, 2004), p. 65.

[52] Z.-L. Liu, J.-S. Sun, R. Li, X.-L. L. Zhang, and L.-C. Cai, Commun. Theor. Phys. 65, 613 (2016).

[53] M. Yokoo, N. Kawai, K. G. Nakamura, and K. I. Kondo, Appl. Phys. Lett. 92, 051901 (2008).

[54] S. M. Rassoulinejad-Mousavi and Y. Zhang, Sci. Rep. 8, 2424 (2018). 